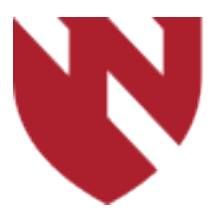

December 2019

\title{
Comprehensive Chronic Pain Treatment: Does Smoking Affect Outcomes?
}

Jamison Hofer

University of Nebraska Medical Center

David Cates

University of Nebraska Medical Center

Valerie K. Shostrom

University of Nebraska Medical Center

Brendan Thelen

Kaiser Permanente, Sacramento, CA

Kimberley Haynes-Henson

University of Nebraska Medical Center

Tell us how you used this information in this short survey.

Follow this and additional works at: https://digitalcommons.unmc.edu/gmerj

Part of the Higher Education Commons, and the Medicine and Health Sciences Commons

\section{Recommended Citation}

Hofer, J., Cates, D., Shostrom, V. K., Thelen, B., , Haynes-Henson, K. Comprehensive Chronic Pain Treatment: Does Smoking Affect Outcomes?. Graduate Medical Education Research Journal. 2019 Dec $13 ; 1(1)$.

https://digitalcommons.unmc.edu/gmerj/vol1/iss1/4

This Original Report is brought to you for free and open access by DigitalCommons@UNMC. It has been accepted for inclusion in Graduate Medical Education Research Journal by an authorized editor of DigitalCommons@UNMC. For more information, please contact digitalcommons@unmc.edu. 


\title{
Comprehensive Chronic Pain Treatment: Does Smoking Affect Outcomes?
}

\begin{abstract}
Abstract

Objective: To determine whether smoking status effects pain and functional outcomes in a chronic pain program.

Methods: A retrospective cohort study of 178 patients treated at the Nebraska Medicine comprehensive Pain Management Program over a five year period was completed. Outcomes measures were the Visual Analog Scale Past Month Average Pain score (VAS-PMA), Multidimensional Pain Inventory pain and interference scales (MPI-P and MPI-I), and the Pain and Impairment Relationship Scale (PAIRS). Patients were categorized by smoking status into non-smoker or current smoker groups. Wilcoxon tests were used to compare the pre scores, post scores, and post-minus-pre scores between smoking status groups.

Results: The pre-treatment VAS-PMA and MPI-P median scores were significantly higher in the current smoker group (81.5 and 5.0, respectively) compared to the non-smoker group (76.5 and 4.3, respectively), whereas post-treatment median scores did not differ. Furthermore, the current smoker group had a significantly greater decrease on the MPI-P from pre- to post-treatment (median=-2.0) than the nonsmoker group (median=-1.6). In addition, smokers had a significantly higher pre-treatment PAIRS score (73.5) than nonsmokers (70), whereas post-treatment scores did not differ.
\end{abstract}

Conclusion: Smokers and non-smokers both benefit from the program, but smokers, who report greater initial pain and stronger beliefs about the association between pain and functional impairment, may benefit more than non-smokers.

\section{Keywords}

Chronic Pain Programs, Smoking, Chronic pain

\section{Creative Commons License}

\section{(c) $(1) \Theta \Theta$}

This work is licensed under a Creative Commons Attribution-Noncommercial-No Derivative Works 4.0 License. 


\section{Comprehensive Chronic Pain Treatment: Does Smoking Affect Outcomes? \\ Jamison Hofer ${ }^{1}$, David Cates ${ }^{2}$, Valerie K. Shostrom ${ }^{3}$, Brendan Thelen ${ }^{4}$, Kimberley Haynes-Henson ${ }^{5}$}

${ }^{1}$ University of Nebraska Medical Center, Department of Neurology

${ }^{2}$ University of Nebraska Medical Center, Department of Psychiatry

${ }^{3}$ University of Nebraska Medical Center, College of Public Health, Department of Biostatistics

${ }^{4}$ Kaiser Permanente, Sacramento, CA

${ }^{5}$ University of Nebraska Medical Center, Department of Anesthesiology

https://doi.org/10.32873/unmc.dc.gmerj.1.1.004

\section{Abstract}

Introduction: Comprehensive pain programs are important for helping patients who have found little relief for their pain with other treatment modalities. It is important to know what factors affect the success of the program and who should be referred to the program. This study seeks to determine whether smoking status affects pain and functional outcomes in a chronic pain program.

Methods: A retrospective cohort study of 178 patients treated at the Nebraska Medicine comprehensive Pain Management Program over a five-year period was completed. Outcome measures were the Visual Analog Scale Past Month Average Pain score (VASPMA), Multidimensional Pain Inventory pain and interference scales (MPI-P and MPI-I), and the Pain and Impairment Relationship Scale (PAIRS). Patients were categorized by smoking status into non-smoker or current smoker groups. Wilcoxon tests were used to compare the pre-scores, post-scores, and post-minus-pre scores between smoking status groups.

Results: The pre-treatment VAS-PMA and MPI-P median scores were significantly higher in the current smoker group (81.5 and 5.0 , respectively) compared to the non-smoker group (76.5 and 4.3, respectively), whereas post-treatment median scores did not differ. Furthermore, the current smoker group had a significantly greater decrease on the MPI-P from pre- to post-treatment (median $=-2.0$ ) than the non-smoker group (median=-1.6). In addition, smokers had a significantly higher pre-treatment PAIRS score (73.5) than nonsmokers (70), whereas post-treatment scores did not differ.

Conclusion: Smokers and non-smokers both benefit from the program, but smokers, who report greater initial pain and stronger beliefs about the association between pain and functional impairment, may benefit more than non-smokers.

\section{Introduction}

Patients with chronic pain often fail to find relief with multiple types of medical and surgical interventions and may be referred to comprehensive pain programs (CPP). These intensive programs offer a multidisciplinary approach that enables patients to better manage their pain using psychological, behavioral, and physical therapy techniques. Treatment typically involves meeting individually and in groups with psychologists, physical therapists, nurses, and physicians to learn strategies to maximize function and better cope with pain. To date, there have been few studies of patient factors that correlate with CPP success. ${ }^{1,2}$ However, smoking and its relationship to chronic pain has long been studied, with smokers often reporting a greater number of painful sites and pain intensity compared to non-smokers. ${ }^{1,3}$ It is not known whether smoking status is associated with outcomes in CPPs. One prior study evaluated the relationship between smoking status and outcomes among patients who completed a CPP, finding a negative association between smoking and employment status after the program. ${ }^{4}$ There is a need for more data regarding the impact of smoking on CPP outcomes. A retrospective cohort study was performed analyzing outcomes of the Nebraska Medicine comprehensive Pain Management Program over a five year period, to identify if smoking status correlated with treatment outcomes as measured by the Visual Analog Scale (VAS), Multidimensional Pain Inventory (MPI), and Pain and Impairment Relationship Scale (PAIRS). ${ }^{5,6,7}$

\section{Methods}

Measures: Visual Analog Scale (VAS). The VAS is a series of 10-centimeter lines with descriptive anchors at each end ("No pain" and "Pain at its worst") on which patients are asked to mark an " $\mathrm{X}$ " to represent their average level of pain on a good day, bad day, today, and over the past month, respectively. The past month average pain level was identified, a priori, for use in the current study. ${ }^{6}$

Multidimensional Pain Inventory (MPI). The MPI is a 61-item questionnaire with 13 subscales assessing the psychosocial impact of chronic pain. Two subscales were identified a priori for use in this study, namely the "Pain" scale, to measure pain intensity and pain-related suffering, and the "Interference" scale to measure interference of pain in relationships and daily activities. ${ }^{7}$

Pain and Impairment Relationship Scale (PAIRS). The PAIRS is a 15 -item questionnaire measuring the extent to which patients believe their pain is associated with their functional status or impairment. Each item consists of a statement followed by a 7-point Likert scale on which patients indicate their level of agreement or disagreement with the statement (e.g., "I have come to accept that I am a disabled person due to my chronic pain."). ${ }^{5}$

Procedures: The Nebraska Medicine Pain Management Program is a multidisciplinary, four-week, day-long treatment program, designed to help individuals with chronic non-malignant pain. Services are provided in a multidisciplinary format by clinical psychologists, physical therapists, a nurse, and a pain physician. Assessment and treatment services are comprehensive and integrated. The primary purpose of the program is to help patients cope more effectively with pain-related problems and maximize their functioning. Specifically, treatment involves three components, which occur simultaneously: Physical therapy, psychological treatment, and medication program/nursing, with the latter involving a physician-monitored opioid tapering program for patients who enter the program on opiates.

Admission criteria include pain that is chronic and non-malignant; desire for non-invasive pain treatment; stability of other medical problems; no psychiatric hospitalizations, suicide attempts, self-harm or harm to others in the last three months; involvement of a family member or significant other for a one-time meeting; willingness to complete an opiate medication taper with the goal of remaining off opiate pain medication in the long term; free of substance abuse (illicit drugs, alcohol, misuse of prescription medications); memory and concentration adequate to benefit from classroom lectures; 5 minute walking tolerance; and ability to complete a floor to standing transfer independently. Patients may be referred by a primary care or specialty physician, or may be self-referred. Patients were not encouraged to quit smoking as part of the program, due 
to the additional stress this would entail beyond that associated with the program itself. However, for those motivated to quit, the program helped develop a post- discharge plan for smoking cessation.

Pre- and post-intervention scores on the Visual Analog Scale past month average (VASPMA), Multidimensional Pain Inventory pain and interference Scales (MPI-P, MPI-I), and Pain and Impairment Relationship Scale (PAIRS) were analyzed for patients over the age of 19-years old who participated in the Nebraska Medicine CPP from 2010 - 2015. Institutional Review Board (IRB) approval was obtained for the research. Smoking status was determined via chart review as part of a previous study. ${ }^{2}$ Former smokers were excluded as the duration of time since they had quit smoking was not established.

PC SAS version 9.4 software was used for all analyses. Only subjects with complete data (pre- and post- values on all paired data) were included in the analyses. Demographic variables, including gender, race, and marital status, were examined for association with smoking status using Chi-Square tests or Fisher's Exact Tests when cell counts were low. Outcome measures were tested for normality using the Wilks-Shapiro test. None of the outcomes passed the test of normality; thus, nonparametric Wilcoxon tests were used to compare the pre- scores, post- scores, and post-minus-pre scores between smoking status groups. The median and quartiles are presented. The outcome "program completed" was compared between smoking groups using a Fisher's Exact Test.

\section{Results}

Tables 1 and 2 show basic demographic data. A total of 178 patients were categorized by smoking status into non-smokers $(\mathrm{N}=120)$ and current smokers $(\mathrm{N}=58)$. The overall population was $70 \%$ female and $30 \%$ male, with $81 \%$ white and $19 \%$ non-white or unknown. Table 1 shows the mean age of the sample was 43.6 years, with a standard deviation of 12.88 years. Table 2 contains descriptive statistics and Chi-Square or Fisher's Exact Test p-values for the comparison of smokers versus non-smokers on each of the demographic variables. None of the differences were statistically significant.

Table 3 shows descriptive statistics (median and quartiles) and Wilcoxon p-values for the pre-treatment, post-treatment, and postminus-pre VAS-PMA scores for current smokers versus non-smokers. The pretreatment VAS-PMA median was significantly higher in the current smoker group (81.5) compared to the non-smoker group (76.5). However, the post-treatment VAS-PMA was not significantly different between current smokers (median=52) and non-smokers (median=53). The median difference (post pre) was not statistically significant between the two groups.

Table 4 contains descriptive statistics (median and quartiles) and Wilcoxon p-values for the pre-treatment, post-treatment, and post-minus-pre MPI-P scores for current smokers versus non-smokers. Pre-treatment MPI-P scores were significantly higher in the current smoker group (median=5.0) versus the non-smoker group (median=4.3). The post-treatment median MPI-P score was not significantly different between smoking status groups. The current smoker group had a significantly greater decrease from pre- to post-treatment (median=-2.0) than the nonsmoker group (median=-1.6).

Table 5 shows descriptive statistics (median and quartiles) and Wilcoxon p-values for the pre-treatment, post-treatment, and post-minuspre MPI-I scores for current smokers versus non-smokers. None of the differences were statistically significant.

Table 6 contains descriptive statistics (median and quartiles) and Wilcoxon p-values for the pre-treatment, post-treatment, and postminus-pre PAIRS scores for current smokers versus non-smokers. Pre-treatment PAIRS scores were significantly higher in the current smoker group (median=73.5) versus the nonsmoker group (median=70.0). There were no statistically significant differences in the posttreatment PAIRS scores or the post-minus-pre PAIRS scores.

Table 7 shows descriptive statistics for the variable "program completed." Two subjects from the non-smoker group had missing data for this variable and were not included in the summary or analysis. In total, $97 \%$ of current smokers completed the program compared to $99 \%$ of non-smokers. The result was not statistically significant.

Analyses were also performed to see if the changes from pre to post were significant within the smoking and non-smoking groups separately, using Wilcoxon signed rank test. The analyses within the two groups for each variable, including difference between VAS, Pain, Interference, and PAIRS, were all statistically significant in both smoking and non-smoking groups with a p-value less than 0.0001 .

\section{Discussion}

Among the current sample of patients who completed a 4-week CPP, current smokers reported more pain at the start of the program compared to nonsmokers, as revealed by pre-treatment scores on both the VAS-PMA and the MPI-P. This finding is consistent with previous research reporting a greater number of painful sites and higher pain intensity among smokers compared to their non-smoker counterparts. ${ }^{1,3}$ In addition to reporting greater pain intensity prior to treatment, current smokers in this study also reported stronger beliefs about the association between their pain and functional impairment compared to non-smokers, as reflected in the PAIRS. At the end of treatment, however, there was no evidence for a significant difference between groups in reports of pain, nor in the association between pain and impairment.

The findings suggest that the program reduced pain intensity, and the extent to which patients believed their pain was associated with functional status, to the same level for both current smokers and non-smokers. In connection with the significantly higher pre-treatment pain scores as well as greater post-minus-pre MPI-P scores among current smokers compared to non-smokers, the results suggest that not only do current smokers benefit from the CPP but they may receive greater benefit when compared with a nonsmoking cohort.

\section{Conclusions}

The Nebraska Medicine Pain Management Program had a high completion rate from 2010 to 2015. Smokers and non-smokers both appear to benefit from the program, and smokers, who report greater initial pain, may benefit more than non-smokers. Smokers are excluded from some pain treatment modalities; however, the data suggest that smokers should not be excluded from CPPs. Further studies should assess whether adding smoking cessation counseling to CPPs increases the magnitude of successful outcomes. 
Table 1.

Demographics (Gender, Race, Smoking History, Marital Status, Age)

\begin{tabular}{lcc} 
& Frequency & Percent \\
\hline Gender & & \\
Female & 125 & 70.22 \\
Male & 53 & 29.78 \\
\hline
\end{tabular}

\begin{tabular}{lcc}
\hline Race & & \\
Not White/Unknown & 34 & 19.10 \\
White & 144 & 80.90
\end{tabular}

\begin{tabular}{lcc}
\hline Smoking History & & \\
Current Smoker & 58 & 32.58 \\
Non-Smoker & 120 & 67.42 \\
\hline Marital Status & & \\
Divorced/Separated & 31 & 17.61 \\
Married/Cohabitating & 95 & 53.98 \\
Single & 43 & 24.43 \\
Widowed/other/unk & 7 & 3.98 \\
\hline
\end{tabular}

Age

Mean: 43.63

Std Dev: 12.88

\section{References}

1 Orhurhu VJ, Pittelkow TP, Hooten WM. Prevalence of smoking in adults with chronic pain. Tobacco Induced Diseases. 2015; 13(1), 17.

2 Thelen B, Cates D, Therrien S, et al. Multidisciplinary treatment of chronic pain patients: Does obesity affect treatment outcome? Poster presented at the International Anesthesia Research Society Annual Meeting and International Science Symposium, Honolulu, HI, 2015.

3 Vogt MT, Hanscom B, Lauerman WC, et al. Influence of smoking on the health status of spinal patients: the National Spine Network database. Spine. 2002; 27(3), 313-319.

4 Fishbain DA, Lewis JE, Cutler R, et al. Does smoking status affect multidisciplinary pain facility treatment outcome? Pain Medicine 2008; 9(8), 1081-1090.

5 Guck TP, Fleischer TD, Willcockson JC, et al. Predictive validity of the pain and impairment relationship scale in a chronic nonmalignant pain population. Archives of physical medicine and rehabilitation, 1999; 80(1), 91-95.

6 Price DD, McGrath PA, Rafii A, et al. The validation of visual analogue scales as ratio scale measures for chronic and experimental pain. Pain 1983; 17(1), 45-56.

7 Ravani PJ. Multidimensional Pain Inventory: Revised profile classification based on clinical observations in a pain setting (Doctoral dissertation) 2005.
Table 2.

Demographic variables N (\%) and Chi-Square or Fisher's Exact Test p-values.

\begin{tabular}{lccc} 
Variable & $\begin{array}{c}\text { Current Smoker } \\
\mathbf{N = 5 8}\end{array}$ & $\begin{array}{c}\text { Non-Smoker } \\
\mathbf{N = 1 2 0}\end{array}$ & P-value \\
\hline Gender (female) & $38(66 \%)$ & $87(73 \%)$ & 0.3396 \\
\hline Race (white) & $45(78 \%)$ & $99(83 \%)$ & 0.4344 \\
\hline Marital Status & & & \\
Divorced/separated & $12(21 \%)$ & $19(16 \%)$ & 0.8052 \\
Married/cohabiting & $28(50 \%)$ & $67(56 \%)$ & \\
Single & $14(25 \%)$ & $29(24 \%)$ & $5(4 \%)$ \\
Widowed/other/UNK & $2(4 \%)$ & & \\
\hline
\end{tabular}

Table 3.

VAS-PMA (Visual Analog Scale past month average) median (quartiles) and Wilcoxon p-values.

\begin{tabular}{lccc} 
Variable & $\begin{array}{c}\text { Current Smoker } \\
\mathbf{N = 5 8}\end{array}$ & $\begin{array}{c}\text { Non-Smoker } \\
\mathbf{N = 1 2 0}\end{array}$ & P-value \\
\hline Pre VAS-PMA & $81.5(73,92)$ & $76.5(66,88)$ & 0.0230 \\
\hline Post VAS-PMA & $52(23,75)$ & $53(35,68)$ & 0.7812 \\
\hline Post-Pre VAS-PMA & $-27(-51,-7)$ & $-23(-39,-9)$ & 0.4028 \\
\hline
\end{tabular}

Table 4.

Multidimensional Pain Inventory pain scale (MPI-P) median (quartiles) and Wilcoxon p-values.

\begin{tabular}{lccc} 
Variable & $\begin{array}{c}\text { Current Smoker } \\
\mathbf{N = 5 8}\end{array}$ & $\begin{array}{c}\text { Non-Smoker } \\
\mathbf{N = 1 2 0}\end{array}$ & P-value \\
\hline Pre MPI-P & $5.0(4.0,5.3)$ & $4.3(4.0,5.0)$ & 0.0214 \\
\hline Post MPI-P & $2.5(1.7,3.7)$ & $2.7(2.0,3.7)$ & 0.3886 \\
\hline Post-Pre MPI-P & $-2.0(-3.0,-1.0)$ & $-1.6(-2.3,-0.7)$ & 0.0362 \\
\hline
\end{tabular}

Table 5.

Multidimensional Pain Inventory interference scale (MPI-I) median (quartiles) and Wilcoxon p-values.

\begin{tabular}{llll} 
Variable & $\begin{array}{c}\text { Current Smoker } \\
\mathbf{N = 5 8}\end{array}$ & $\begin{array}{c}\text { Non-Smoker } \\
\mathbf{N = 1 2 0}\end{array}$ & P-value \\
\hline Pre MPI-I & $4.95(4.50,5.50)$ & $4.80(4.15,5.40)$ & 0.1264 \\
\hline Post MPI-I & $3.20(1.80,4.10)$ & $3.20(2.20,4.10)$ & 0.9122 \\
\hline Post-Pre MPI-I & $-1.90(-2.60,-0.70)$ & $-1.30(-2.30,-0.75)$ & 0.2278 \\
\hline
\end{tabular}

Table 6.

Pain and Impairment Relationship Scale (PAIRS) median (quartiles) and Wilcoxon p-values.

\begin{tabular}{llll} 
Variable & $\begin{array}{c}\text { Current Smoker } \\
\mathbf{N}=\mathbf{5 8}\end{array}$ & $\begin{array}{c}\text { Non-Smoker } \\
\mathbf{N = 1 2 0}\end{array}$ & P-value \\
\hline Pre PAIRS & $73.5(67.0,84.0)$ & $70.0(63.0,79.0)$ & 0.0386 \\
\hline Post PAIRS & $45.5(34.0,63.0)$ & $46.0(37.5,55.5)$ & 0.9629 \\
\hline Post-Pre PAIRS & $-26.0(-40.0,-15.0)$ & $-23.0(-35.0,-14.0)$ & 0.2961 \\
\hline
\end{tabular}

Table 7.

Program Completed N (\%) and Fisher's Exact Test p-value

\begin{tabular}{lccc} 
Variable & $\begin{array}{c}\text { Current Smoker } \\
\mathbf{N}=\mathbf{5 8}\end{array}$ & $\begin{array}{c}\text { Non-Smoker } \\
\mathbf{N}=\mathbf{1 2 0}\end{array}$ & P-value \\
\hline Program Completed & $56(97 \%)$ & $117(99 \%)$ & 0.2529 \\
\hline
\end{tabular}

